\section{OHF APPROVES BIOMIN FOR RELIEF OF TOOTH SENSITIVITY AND REMINERALISATION}

Surveys indicate that more than $40 \%$ of adults experience tooth sensitivity at some stage during their lifetimes. BioMin F, the pioneering toothpaste which replaces lost tooth mineral, has become the first toothpaste to gain accreditation from the Oral Health Foundation (OHF) expert panel for both sensitivity reduction and remineralisation.

BioMin F toothpaste works through its slow release of calcium, phosphate and fluoride ions which form a protective fluoro apatite layer on the tooth surface. This apatite

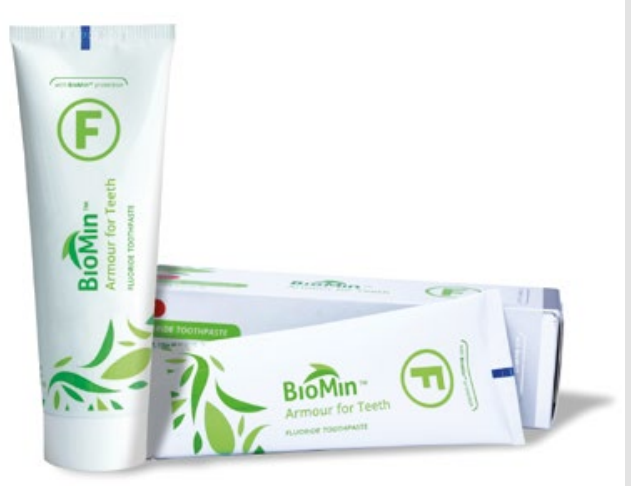

effectively seals any exposed and open dentine tubules which are a key cause tooth sensitivity due to their direct link with the central nervous system.

BioMin Chief Executive Richard Whatley said: 'Oral Health Foundation accreditation is a hugely important endorsement of our toothpaste as it assures consumers that BioMin F has been evaluated by an independent panel of internationally recognised dental experts and academics. These experts have studied all the claims carefully to ensure they are true and are backed up by reliable scientific evidence. OHF has the biggest oral healthcare product accreditation scheme in the world and is internationally recognised. Consumers can be assured that a product which carries the Oral Health Foundation approved logo has had its claims rigorously checked and effectively verified. The accreditation is applicable worldwide.

For more information, visit https:// biomin.co.uk/products/biomintm-ftoothpaste.

\section{PERFECT PROPHYLAXIS}

Lunos, from Durr

Dental, is a

premium prophylaxis brand covering a range of products that offer unique advantages over existing products.

One example of this is the MyLunos powder jet handpiece. Its unique exchangeable chamber principle means the powder container can be replaced quickly and easily, avoiding the inconvenience of having to refill in the middle of treatment. Furthermore, surgeries can prepare enough powder containers for the whole day. The tip can be rotated 360 degrees, which makes it easy for the operator to use as they can access all areas of the oral cavity. All components are autoclavable and the unit fits all standard turbine couplings.

MyLunos works with various prophylaxis powders. The Gentle Clean variant of Lunos ${ }^{\oplus}$ contains innovative new abrasive agents based on the non-carcinogenic disaccharide trehalose for gentle cleaning in the supragingival area and is available in three

\section{AWARD FOR ESTELITE}

Estelite Sigma Quick has been awarded the Dental Advisor Top Product Universal Composite for the 10th year running. Featuring Tokuyama's patented RAP monomer and Aesthetic 'Pearl' technology, Estelite Sigma Quick delivers an extended working time yet cures in only 10 seconds. There is also less residual monomer and minimal after cure colour change for long term aesthetic satisfaction.

In addition, Estelite Sigma Quick offers 'miracle' shade matching. Because of its unique Aesthetic 'Pearls' it offers inherent shade mimicking so that, in most cases, just one shade will blend perfectly with the natural teeth, leaving invisible margins and undetectable restorations.

These 'Pearls' also facilitate outstanding ability to polish that lasts, allied to high wear resistance and very low abrasion of the opposing teeth.

More information is available by calling Trycare Ltd on 01274885544 or visiting www.trycare.co.uk/estelite.

\section{SCALING}

NEW HEIGHTS

Periodontal scaling has never been easier or more comfortable than with the $\mathrm{W} \& \mathrm{H}$ range of cost-effective piezo scalers, including the Pyon 2 and the Tigon.

W\&H piezo scalers not only feature a ring of LED lights that offer superior visibility within the oral cavity, but they can also be customised with W\&H's extensive range of high quality tips. These are suitable for a variety of indications, such as prophylaxis, periodontics, endodontics, implant maintenance and restorative work.

Designed for ultimate ease-of-use, the Tigon enables practitioners to increase or reduce the power of the scaler and the flow volume of the coolant. This ensures patients experience nothing but truly comfortable and time-efficient dental care.

More information can be found by visiting www.wh.com/en_uk, calling 01727 874990 or emailing office.uk@wh.com. 